\title{
Update on the clinical utility and optimal use of cefditoren
}

This article was published in the following Dove Press journal:

International Journal of General Medicine

19 May 2012

Number of times this article has been viewed

\author{
José Barberán' \\ Lorenzo Aguilar ${ }^{2}$ \\ María-José Giménez ${ }^{2}$ \\ 'Infectious Diseases Department, \\ Hospital Central de la Defensa \\ Gomez Ulla, ${ }^{2}$ Microbiology \\ Department, School of Medicine, \\ Universidad Complutense de Madrid, \\ Madrid, Spain
}

\begin{abstract}
This article reviews and updates published data on cefditoren. The in vitro activity of cefditoren and its potential pharmacokinetic/pharmacodynamic adequacy to cover emerging resistance phenotypes in the present decade is reviewed. Cefditoren's in vitro activity against most prevalent bacterial respiratory pathogens in the community and its pharmacokinetic/ pharmacodynamic profile suggests a significant role for cefditoren in the treatment of respiratory tract infections. Clinical trials (in acute exacerbations of chronic bronchitis, communityacquired pneumonia, pharyngotonsillitis, and sinusitis) performed during clinical development outside Japan, mainly in adults, are reviewed, together with new clinical studies in the treatment of pharyngotonsillitis, sinusitis, and otitis media in children, mainly in Japan, for efficacy and safety assessment. The results of these studies support the adequacy of cefditoren for the treatment of community-acquired respiratory tract infections with a safety profile similar to previous oral antibiotics. From the data reviewed, it is concluded that cefditoren is an adequate option for the treatment of mild-to-moderate community-acquired respiratory infections, especially in geographical areas with a reported prevalence of phenotypes exhibiting nonsusceptibility to common oral antibiotics.
\end{abstract}

Keywords: acute exacerbations of chronic bronchitis, community-acquired pneumonia, pharyngotonsillitis, sinusitis, otitis media

\section{Introduction: resistance issues and the development of new antibiotics}

Appropriate use of antimicrobials is defined by the World Health Organization as "the cost-effective use of antimicrobials which maximizes clinical and therapeutic effect while minimizing both drug-related toxicity and the development of antimicrobial resistance." Antimicrobial resistance is one of the most urgent health threats people face all around the world ${ }^{2}$ and is emerging to every class of existing antibiotics, affecting environments, hospitals, and the community. ${ }^{3}$

Increasing awareness of optimizing therapy not only involves maximizing therapeutic outcome but also minimizing the risk of resistance emerging during therapy, both in the infecting pathogen and in the normal flora. ${ }^{4}$ While optimizing outcome is directed at the individual patient level, emergence of resistance is an ecological issue and a trade-off between these two objectives is not easy to achieve. ${ }^{4}$ There is a dynamic situation in which the introduction of new antimicrobials necessitated by the emergence of resistance to existing compounds produces new selective pressure that selects new resistances, thus closing the circle. Therefore, resistance selection and diffusion in the community derived from antibiotic consumption requires, in turn, 
new antibiotics. However, a new antibiotic is only needed if it can counter existing resistances and if it possesses limited capability of resistance selection in human microbiota ("ecological effect").

Around $85 \%-90 \%$ of antibiotic consumption occurs in the community, with $80 \%$ of this consumption for the treatment of respiratory tract infections..$^{5}$ The most prevalent bacterial isolates causing respiratory infections in the community (Streptococcus pneumoniae, Streptococcus pyogenes and Haemophilus influenzae) are part of normal flora in humans, with the nasopharynx as the exclusive host. Antibiotic consumption can select resistant populations or subpopulations present in the nasopharynx that can be further transmitted to other individuals.

$S$. pneumoniae is a natural transformable bacterial pathogen showing rapid evolution in response to clinical interventions. Following the introduction of the 7-valent pneumococcal conjugate vaccine (PCV7) for immunization in children, the incidence of invasive pneumococcal disease has declined both in children and adults (reflecting "herd immunity"). ${ }^{6}$ The decrease in the incidence of invasive disease caused by serotypes included in PCV7 runs in parallel with decreases in nonsusceptibility to penicillin (oral) and erythromycin among invasive isolates, from both adults and children, ${ }^{7,8}$ but not among noninvasive isolates such as those from middle-ear fluid. ${ }^{9}$ However, emergence of serotypes not encompassed by the vaccine is worrisome and may be associated with heightened antimicrobial resistance and virulence. ${ }^{6}$ In Spain, recent published data show that nonsusceptibility rates to penicillin/erythromycin are around 20\% among invasive isolates ${ }^{8}$ but around $45 \%-50 \%$ among middle-ear isolates. ${ }^{9}$ Worldwide, approximately $30 \%$ of isolates are resistant to macrolides ${ }^{10}$ and a similar percentage of $S$. pneumoniae are now considered multidrug resistant. ${ }^{11}$ In contrast, rates of resistance to fluoroquinolones are still low. ${ }^{12}$

However, antibiotic pressure does not always result in the emergence of resistance. In the case of $S$. pyogenes, consumption of $\beta$-lactams has not resulted in the appearance of penicillin resistance, probably because of the absence of selectable variants carrying mechanisms of $\beta$-lactam resistance. ${ }^{13}$ In contrast, the rate of erythromycin resistance varies among countries, ranging from $6.9 \%$ in the USA ${ }^{14}$ to $25.6 \%$ in Hong Kong. ${ }^{15}$ In a recent study performed in Eastern Europe, the rate of erythromycin resistance in $S$. pyogenes was low $(<10 \%)$ in Romania and Baltic countries, intermediate (10\%-20\%) in Poland and the Czech Republic, and high $(>25 \%)$ in Hungary and Slovakia. ${ }^{16}$ In Spain, the erythromycin resistance rate was $19.0 \%$ in $2006-2007 .{ }^{17}$
In 2000, the first fluoroquinolone-resistant $S$. pyogenes isolate was reported; ${ }^{18}$ since then, reports of such isolates have occurred in the USA, Europe, and Japan. ${ }^{19-22}$

Haemophilus influenzae resistance to fluoroquinolones continues to be exceptionally rare; however, this species is intrinsically resistant to macrolides, which is associated with the presence of efflux pumps in virtually all strains. ${ }^{23}$ Ampicillin resistance varies on a geographical and temporal basis, from $8.7 \%$ in South Africa to approximately 30\% in $\mathrm{Asia}^{24}$ and the USA. ${ }^{25}$ In Europe, mean ampicillin resistance has been reported as $16.4 \%$, with resistance due to $\beta$-lactamase production ranging from $17.6 \%$ in France to $0 \%$ in Germany and The Netherlands. ${ }^{26}$ With respect to strains showing mutations in the ftsI gene encoding PBP3 ( $\beta$-lactamase-negative ampicillin-resistant [BLNAR] strains and $\beta$-lactamase positive amoxicillin/clavulanate-resistant [BLPACR] strains), one study carried out in Europe and Canada in 2006-2007 showed an 11.4\% prevalence of genotypic BLNAR. ${ }^{27}$ The country with the highest prevalence was Japan, with $42.9 \%$ BLNAR $^{28}$ and an increase in the prevalence of BLPACR from 1999 to $2008 .^{29}$

\section{In vitro activity and pharmacokinetics/ pharmacodynamics of cefditoren}

The intrinsic activity of cefditoren against $S$. pneumoniae isolates has been explored in different studies. ${ }^{30-41}$ Cefditoren showed high intrinsic activity against penicillin-susceptible strains (minimum inhibitory concentration $[\mathrm{MIC}]_{90}$ from $\leq 0.03$ to $0.06 \mu \mathrm{g} / \mathrm{mL}$ ), with $\mathrm{MIC}_{90}$ ranging from 0.25 to $0.5 \mu \mathrm{g} / \mathrm{mL}$ against penicillin-intermediate and from 0.5 to $1 \mu \mathrm{g} / \mathrm{mL}$ against penicillin-resistant isolates. MIC values against penicillin-intermediate and -resistant strains were lower than those of amoxicillin, cefdinir, cefprozil, cefuroxime, cefixime, ceftibuten, cefpodoxime, erythromycin, clarithromycin, and azithromycin, ${ }^{32-41}$ with the $\mathrm{MIC}_{90}$ of cefditoren against penicillin nonsusceptible isolates one-dilution lower than that of cefotaxime. In the most recently published surveillance, the $\mathrm{MIC}_{90}$ of cefditoren was similar to that of ceftriaxone. ${ }^{42}$

Against S. pyogenes and $H$. influenzae, cefditoren has demonstrated markedly high intrinsic activity, with an $\mathrm{MIC}_{90}$ of $\leq 0.06 \mu \mathrm{g} / \mathrm{mL}$ in the studies performed. ${ }^{16,17,32,34,36,41,43-47} \mathrm{In}$ the case of $H$. influenzae, the high intrinsic activity of cefditoren was maintained against strains exhibiting $f$ tsI mutations in contrast to amoxicillin/clavulanate and cefuroxime. . $3,45,46$

Cefditoren is orally administered as cefditoren-pivoxil, which is further hydrolyzed during absorption and distributed in the blood as active cefditoren. In fasting patients, the oral 
bioavailability of cefditoren-pivoxil is low $(15 \%-20 \%)$, but when administered with high-fat meals, the mean maximum concentration $\left(\mathrm{C}_{\max }\right)$ and area under the concentration-time curve (AUC) values for cefditoren increase to $50 \%$ and $70 \%$, respectively. ${ }^{48}$ In a Phase I study administering a single $400 \mathrm{mg}$ dose of cefditorenpivoxil with a high-fat meal to Caucasian male subjects $\geq 18$ years of age, values of the pharmacokinetic parameters determined were: $\mathrm{C}_{\max }=3.7 \pm 0.7 \mu \mathrm{g} / \mathrm{mL}$, time to achieve maximum concentration in serum $\left(\mathrm{T}_{\max }\right)=2 \mathrm{~h}, \mathrm{AUC}_{0-\infty}=12.5 \pm 1.6 \mu \mathrm{g} \times \mathrm{h} /$ $\mathrm{mL}$ and half-life time to achieve $\mathrm{C}_{\text {max }}\left(\mathrm{T}_{\text {max }}\right)\left(\mathrm{t}_{1 / 2}\right)=1.54 \pm 0.20$ $\mathrm{h} .{ }^{49}$ Binding to plasma proteins (primarily human serum albumin) averages $88 \%$ for cefditoren. ${ }^{48}$

As for all $\beta$-lactams, the pharmacokinetic/pharmacodynamic parameter predicting bacterial eradication and subsequent efficacy for cefditoren is the time (expressed as \% dosing interval) that serum concentrations exceed the MIC of the infecting pathogen $(\% \mathrm{t}>\mathrm{MIC})$. Values of $33 \% \mathrm{t}>\mathrm{MIC}$ are considered bacteriostatic endpoints ${ }^{50}$ and those of $40 \%$ $\mathrm{t}>\mathrm{MIC}$ as predictive values for clinical cure in humans. ${ }^{51,52}$ To investigate the pharmacodynamic coverage of cefditoren, a Monte Carlo simulation was performed ${ }^{53}$ using data from the mentioned Phase I study in ten healthy male volunteers, where $400 \mathrm{mg}$ of cefditoren-pivoxil was administered orally as single dose after a high-fat meal. ${ }^{49}$ Cut-off values considered were $33 \% \mathrm{t}>\mathrm{MIC}$ and $40 \% \mathrm{t}>\mathrm{MIC}$. Target attainments $>90 \%$ were obtained for MICs of $0.5 \mu \mathrm{g} / \mathrm{mL}$ for total drug (using both cut-off values) and $0.25 \mu \mathrm{g} / \mathrm{mL}$ $(33 \% \mathrm{t}>\mathrm{MIC})$ and $0.12 \mu \mathrm{g} / \mathrm{mL}(40 \% \mathrm{t}>\mathrm{MIC})$ for the free drug. ${ }^{53}$ This means that, using the strictest cut-off ( $40 \%$ $\mathrm{t}>\mathrm{MIC}$ ), concentrations obtained after administration of $400 \mathrm{mg}$ cefditoren-pivoxil cover isolates with MICs up to $0.5 \mu \mathrm{g} / \mathrm{mL}$ (total drug) and up to $0.12 \mu \mathrm{g} / \mathrm{mL}$ (free drug), with a probability of $\geq 96 \%$. ${ }^{53}$ Taking advantage of the fact that cefditoren protein-binding rates in mice and humans are almost identical, study of a pneumococcal sepsis mouse model was undertaken. Animals were infected with penicillinintermediate and -resistant isolates (MICs of 1 and $2 \mu \mathrm{g} / \mathrm{mL}$ for penicillin and cefditoren, respectively) and $100 \%$ survival rates (vs $0 \%$ in untreated controls) were linked to cefditoren $\mathrm{t}>\mathrm{MIC} \approx 35 \%$ (free $\mathrm{t}>\mathrm{MIC} \approx 20 \%),{ }^{54}$ values lower than those classically considered for therapeutic efficacy.

\section{Clinical studies of efficacy Pharyngotonsillitis}

Several studies ${ }^{55-59}$ have been published on cefditoren in recent years, providing additional information to data obtained from clinical trials performed during the clinical development of cefditoren. ${ }^{60-62}$
The most recently published study, by Tsumura et al compared cefditoren-pivoxil for 5 days versus amoxicillin for 10 days in children with group A beta-hemolytic streptococci acute tonsillopharyngitis in Japan. ${ }^{55}$ No difference in clinical efficacy was found between both arms $(100 \%$ for cefditoren vs $97.9 \%$ for amoxicillin), with $100 \%$ bacteriological efficacy in both groups. ${ }^{55}$ Changes in oral microflora were also assessed, showing absence of flora disturbance with cefditoren in contrast with amoxicillin, which produced a clear decrease in oral microbial flora. ${ }^{55}$

The same comparator was used in the study by Ozaki et al where children were randomized to receive either $3 \mathrm{mg} / \mathrm{kg}$ cefditoren-pivoxil three times daily (tid) for 5 days (103 patients; mean age: $5.5 \pm 2.3$ years) or amoxicillin $10 \mathrm{mg} / \mathrm{kg}$ tid for 10 days (155 patients; mean age: $5.2 \pm 2.0$ years).$^{56}$ The 258 isolates recovered showed $\mathrm{MIC}_{90}$ values of $0.06 \mu \mathrm{g} / \mathrm{mL}$ for amoxicillin and $\leq 0.03 \mu \mathrm{g} / \mathrm{mL}$ for cefditoren. ${ }^{56}$ Eradication was $100 \%$ with amoxicillin and $99 \%$ with cefditoren-pivoxil, without differences in recurrence rates during the 4-week follow-up period: $9.7 \%$ in the amoxicillin arm and $7.8 \%$ in the cefditoren-pivoxil arm. ${ }^{56}$

In a different open prospective multicenter study by Sakata, cefditoren-pivoxil (3 $\mathrm{mg} / \mathrm{kg}$ tid) was administered for 7 days to 90 enrolled children (age range: 8 months to 12 years).$^{57} \mathrm{~A}$ total of 79 patients complied with treatment intake and follow-up visits (at the end of therapy and 1 month after end of therapy) and were included in the efficacy analysis. Isolates of the same basal $\mathrm{T}$ type were found at the end of therapy in four patients and five patients presented with recurrence 1 month after the end of therapy (again with the same $\mathrm{T}$ type and pulsed field gel electrophoresis pattern). ${ }^{57}$

Kawamata et al evaluated the efficacy of cefditoren in an open multicenter study (147 centers) including a large number of children presenting with $S$. pyogenes laryngopharyngitis (464 patients) or tonsillitis (254 patients). ${ }^{58}$ Age range was 9 months to 14 years (median age: 5 years) and mean daily doses were $\geq 9 \mathrm{mg} / \mathrm{kg}$ and $<13.5 \mathrm{mg} / \mathrm{kg} .{ }^{58}$ The clinical response rate was $98.5 \%$ for laryngopharyngitis and $98.4 \%$ for tonsillitis. ${ }^{58}$ In the subset of patients in which bacteriological response could be assessed (205 for laryngopharyngitis and 119 for tonsillitis), the $S$. pyogenes eradication rate was $94.6 \%$ and $92.4 \%$, respectively. ${ }^{58}$

In two of these three studies performed in Japan, ${ }^{56,57}$ the bacteriological recurrence rate ranged from $6.3 \%$ to $9.7 \%$ in the treatment of $S$. pyogenes pharyngotonsillitis with $\beta$-lactams. It has been reported that clinical isolates from recurrent streptococcal pharyngitis show emm typespecific features, with emm 12 the most frequently detected 
(expressing PrtF1 protein that allows higher invasive capability) followed by emm6 (more likely producing biofilm, thus allowing embedded $S$. pyogenes survival). ${ }^{59}$ In the absence of specific resistance traits, localization of $S$. pyogenes in biofilms or inside tonsillar tissue can contribute to functional antibiotic resistance. ${ }^{63,64}$ In addition, with respect to $\beta$-lactams that can be hydrolyzed by $\beta$-lactamases, the presence of resident bacteria producing these inactivating enzymes (as with $H$. influenzae isolated from adeno-tonsil samples that also produces biofilms ${ }^{65}$ ) and the specific resistance traits in these indirect pathogens have also been proposed as an explanation for treatment failures ${ }^{66}$ and recurrences. ${ }^{67}$

Cefditoren has been assessed in the treatment of pharyngotonsillitis episodes in children presenting recurrent pharyngotonsillitis. The study by Kikuta et al compared the efficacy obtained with $9 \mathrm{mg} / \mathrm{kg}$ tid cefditoren-pivoxil for 5 days versus 10 days in children (mean age: $6.77 \pm 2.04$ years) with a history of at least one previous episode of group A $\beta$-hemolytic streptococcal pharyngotonsillitis. ${ }^{68} \mathrm{~A}$ total of 77 and 149 patients were included in the 5-day and 10-day treatment arms, respectively. ${ }^{68}$ The frequency of previous episodes was $2.79 \pm 1.35 .{ }^{68}$ Bacteriological failure was similar in the 5-day treatment arm (9.1\%) and the 10-day treatment group (11.4\%), but significantly higher recurrences were found in the 5 -day treatment group $(9.1 \%$ vs $0.7 \% ; P=0.03) .{ }^{68}$ The interval between the preceding episode and the presently treated episode was lower $(P=0.018)$ in the treatment failure group $(7.05 \pm 7.31$ months $)$ than in the treatment success group (11.04 \pm 14.57 months). ${ }^{68}$ No differences in clinical failure were found between groups: $18.2 \%$ in the 5-day group versus $12.1 \%$ in the 10 -day group. ${ }^{68}$

Efficacy results of all these studies in children carried out in Japan support efficacy data obtained during the clinical development of cefditoren outside Japan in the treatment of pharyngotonsillitis, including that from 1322 randomized patients $\geq 12$ years of age analyzed in an efficacy pooled analysis. ${ }^{62}$ No significant differences were found between the clinical response obtained with cefditoren-pivoxil and penicillin V (95.3\% vs 92.2\% at end of treatment and $91.9 \%$ vs $89.4 \%$ at late follow-up), but eradication of $S$. pyogenes was higher with cefditoren-pivoxil in the two studies with microbiological assessment: ${ }^{60,61} 90.4 \%$ versus $82.7 \%$ $(P=0.002)$ at the end of therapy and $84.7 \%$ versus $76.7 \%$ $(P=0.008)$ at the end of follow-up. ${ }^{62}$ Bacteriological efficacy was associated with higher clinical responses: 98.5\%/99.0\% at the end of treatment/follow-up for cefditoren-pivoxil and $99.3 \% / 98.9 \%$ for penicillin $\mathrm{V}$ among patients showing bacterial eradication, in contrast to clinical responses of $51.4 \% / 32.7 \%$ at the end of treatment/follow-up for cefditoren-pivoxil and $49.2 \% / 41.5 \%$ for penicillin $\mathrm{V}$ in patients showing $S$. pyogenes persistence. ${ }^{62}$

\section{Acute sinusitis}

A recently published study of children in Thailand evaluated the efficacy of cefditoren in the treatment of bacterial rhinosinusitis. ${ }^{69}$ This randomized, investigator-blinded controlled study in children aged 1-15 years compared the clinical efficacy provided by cefditoren-pivoxil 4-6 mg/kg twice per day (bid) (maximum dose $300 \mathrm{mg} /$ day) versus 80-90 mg/kg/day amoxicillin amoxicillin/clavulanic acid (maximum dose $800 \mathrm{mg} /$ day) administered bid for 14 days. ${ }^{69}$ Changes in sinus symptoms were assessed daily by patients or parents using a quantitative symptom score. The primary outcome was rate of improvement 7 and 14 days after the initial visit and the secondary outcomes were relapse (defined as subjective rating of lack of improvement at day 21 or 28 in a patient rated as improved on day 14), recurrence (defined as sinus symptoms lasting for $\geq 10$ days during the second month of follow-up in a patient rated as improved on day 28), and time to improvement. ${ }^{69} \mathrm{~A}$ total of 66 patients were evaluated in the cefditoren-pivoxil group and 72 in the amoxicillin/ clavulanic acid. ${ }^{69}$ The median time to improvement was 3.0 days in both groups, without differences in rates of improvement between groups: $78.8 \%$ for cefditoren-pivoxil versus $84.7 \%$ for amoxicillin/clavulanic acid. The differences in rates of relapse at day $28(9.1 \%$ for cefditoren-pivoxil vs $11.1 \%$ for amoxicillin/clavulanic acid) and recurrence rates $(3.0 \%$ for cefditoren-pivoxil vs $5.6 \%$ for amoxicillin/ clavulanic acid) were not statistically significant. ${ }^{69}$

Efficacy results from children obtained in this study are in accordance with efficacy rates obtained in adults in the clinical trials performed during the clinical development of cefditoren outside Japan. ${ }^{62,70}$ Data from the three clinical trials were analyzed in a pooled analysis. ${ }^{62}$ Regimens compared were cefditoren-pivoxil $200 \mathrm{mg}$ or $400 \mathrm{mg}$ bid for 10 days, cefuroxime-axetil $250 \mathrm{mg}$ bid for 10 days, and amoxicillin/ clavulanic acid $875 / 125 \mathrm{mg}$ bid for 10 days or $500 / 125 \mathrm{mg}$ tid for 10 days. Clinical response was considered resolution or improvement in all pretreatment signs/symptoms with at least no worsening in the radiographic appearance of the sinus without the need for additional therapy. Patients were assessed pretherapy, at the end of treatment (within 48 hours of the last dose intake) and at the end of follow-up (7-14 days in one study and $34 \pm 2$ days after the last dose intake in the two remaining studies). ${ }^{62} \mathrm{~A}$ total of 1819 randomized patients 
(mean age: $39.8 \pm 14.3$ years) were included: 1726 patients in the intention-to-treat and 1589 patients in the per-protocol populations. ${ }^{62}$ No differences $(P>0.001)$ in clinical response were found by comparing cefditoren-pivoxil $200 \mathrm{mg}$ versus cefditoren-pivoxil $400 \mathrm{mg}$ versus comparators both at the end of therapy $(81.1 \%$ vs $80.2 \%$ vs $84.8 \%)$ and at the end of follow-up (72.1\% vs $71.2 \%$ vs $77.4 \%){ }^{62}$

\section{Acute otitis media}

No trials in the treatment of acute otitis media were performed during the clinical development of cefditoren outside Japan. A limited number of clinical studies have been published in Japan using cefditoren for the treatment of acute otitis media in children. ${ }^{71-74}$ The most recently published study corresponds to a huge postmarketing study in pediatric patients with acute otitis media performed in 305 medical institutions with 2006 patients. ${ }^{71}$ Age range was 1 month-14 years (median: 3 years) and up to $90 \%$ of patients presented with moderate/severe acute otitis media. ${ }^{71}$ The median daily dose of cefditoren was $10.0 \mathrm{mg} / \mathrm{kg}$ and median total treatment period was 7 days. A total of 1958 patients were eligible as efficacy analysis population, with a clinical response rate of $93.5 \%{ }^{71}$ In 832 patients, the causative organism was detected during the baseline microbiological examination, with 1217 isolates identified. ${ }^{71}$ S. pneumoniae $(\mathrm{n}=397)$, H. influenzae $(\mathrm{n}=393)$, and Moraxella catarrhalis $(\mathrm{n}=166)$ together represented up to $78.6 \%$ of all isolates and 261 microorganisms were from other species. ${ }^{71}$ Among the 397 isolated S. pneumoniae strains, $58.9 \%$ were nonsusceptible to penicillin and among the 393 H. influenzae isolates, $28.0 \%$ were ampicillin nonsusceptible (mainly BLNAR strains: $27.2 \%$ ). ${ }^{71}$ The per-pathogen clinical response rate was $89.7 \%$ for $S$. pneumoniae, $90.3 \%$ for $H$. influenzae, $92.2 \%$ for M. catarrhalis, and $92.7 \%$ for other microorganisms. ${ }^{71}$ In patients infected by isolates with specific resistance traits, clinical and bacteriological responses were $88 \%$ and $85.7 \%$, respectively, for penicillinintermediate pneumococci; $90.1 \%$ and $77.5 \%$, respectively, for penicillin-resistant pneumococci; and $92.5 \%$ and $81.8 \%$, respectively, for BLNAR $H$. influenzae. ${ }^{71}$

\section{Lower respiratory tract infections}

No further clinical studies have been published with cefditoren in the treatment of lower respiratory tract infections apart from those performed during the clinical development of cefditoren (four studies in community-acquired pneumonia [CAP] and three studies in acute exacerbations of chronic bronchitis $[\mathrm{AECB}])$. Data from these clinical trials were pooled and analyzed by comparing cefditoren with comparators (amoxicillin/clavulanic acid, cefuroxime-axetil, cefpodoxime, and clarithromycin) in a previous publication. ${ }^{75}$ The per-pathogen bacteriological response was determined by pooling data from CAP and AECB studies. Response was defined as eradication or presumed eradication (absence of sputum for culture in a patient with clinical response). ${ }^{75} \mathrm{~A}$ total of 406 S. pneumoniae isolates (56 of them nonsusceptible to oral penicillin) were recovered prior to treatment initiation, with similar bacteriological response rates for cefditoren and comparators (88.5\% for cefditoren-pivoxil $200 \mathrm{mg}, 92.0 \%$ for cefditoren-pivoxil $400 \mathrm{mg}$, and $89.9 \%$ for comparators). ${ }^{75}$ Of the 56 penicillin nonsusceptible (MIC $\geq 0.12 \mu \mathrm{g} / \mathrm{mL}$ ) $S$. pneumoniae isolates recovered, all those isolated from patients in the cefditoren-pivoxil $400 \mathrm{mg}$ group $(\mathrm{n}=20)$, $16 / 19(84.2 \%)$ in the cefditoren-pivoxil $200 \mathrm{mg}$ group, and $16 / 17(94.1 \%)$ in the comparators group were eradicated or presumed eradicated. ${ }^{75} \mathrm{Up}$ to 29 of the 56 nonsusceptible isolates were penicillin resistant ( $\mathrm{MIC} \geq 2 \mu \mathrm{g} / \mathrm{mL}$ ), 18 in the cefditoren-pivoxil arm and eleven in the comparators group. Eradication or presumed eradication rates among patients with penicillin-resistant initial isolates were $94.4 \%$ (17/18) for cefditoren (pooled $200 \mathrm{mg}$ and $400 \mathrm{mg}$ ) and 90.9\% (10/11) for comparators. ${ }^{75} \mathrm{H}$. influenzae was isolated in baseline cultures of 595 patients: 224 patients in the cefditoren-pivoxil $200 \mathrm{mg}$ arm, 175 in the cefditoren-pivoxil $400 \mathrm{mg}$ arm, and 196 in the group of pooled comparators. ${ }^{75}$ Bacteriological response rates were similar in all groups: $86.6 \%$ for cefditoren-pivoxil $200 \mathrm{mg}$ versus $85.7 \%$ for cefditoren-pivoxil $400 \mathrm{mg}$ versus $82.7 \%$ for comparators. ${ }^{75}$

For the clinical response analysis, data from AECB and CAP studies were analyzed separately. Pooled analyses included 1379 patients with CAP and 1560 patients with AECB as the overall efficacy populations. ${ }^{75}$ Similar clinical response rates were obtained for cefditoren-pivoxil $200 \mathrm{mg}$ (91.8\%), cefditoren-pivoxil $400 \mathrm{mg}$ (89.2\%), and comparators $(91.5 \%)$ at the end of therapy and at the end of follow-up (87.8\% vs.85.9\% vs. $90.4 \%$, respectively) in CAP studies. ${ }^{75}$ Similar response rates were found in AECB studies: cefditoren-pivoxil $200 \mathrm{mg}(85.8 \%)$ versus cefditoren-pivoxil $400 \mathrm{mg}(91.3 \%)$ versus comparators $(87.1 \%)$ at the end of therapy and $81.3 \%$ versus $81.2 \%$ versus $83.3 \%$, respectively, at the end of follow-up. ${ }^{75}$ However, slightly higher efficacy was obtained with $400 \mathrm{mg}$ cefditoren-pivoxil (vs $200 \mathrm{mg}$ ) by the end of therapy $(91.3 \%$ vs $85.8 \%, P=0.014) .{ }^{75}$

\section{Safety and tolerability issues}

Safety data from 13 clinical trials carried out in adults during the clinical development of cefditoren outside Japan for the 
treatment of community-acquired respiratory infections were analyzed in a pooled analysis and showed that the adverse event profiles of cefditoren-pivoxil and comparators are similar. ${ }^{76}$ For both groups, diarrhea was the most frequent adverse event (9.9\% for cefditoren vs $6.9 \%$ for comparators) followed by vaginosis among female populations $(3.9 \%$ vs $4.6 \%)$, nausea $(3.5 \%$ vs $3.6 \%)$, abdominal pain $(1.8 \%$ vs $1.1 \%)$, and dyspepsia (1.1\% vs $0.9 \%){ }^{76}$

New data from recently published studies update the information mainly with data from children in Japan. In a postmarketing surveillance evaluating safety in 2006 children with acute otitis media treated with cefditoren-pivoxil (median daily dose: $10.0 \mathrm{mg} / \mathrm{kg}$ with a median total treatment period of 7 days), the incidence of adverse reactions was $1.79 \%$, without unexpected or serious adverse drug reactions reported..$^{71}$ The most frequent adverse drug reaction was diarrhea $(1.30 \%)$ that resolved or subsided during cefditoren-pivoxil treatment or after discontinuation or completion of therapy in all cases. ${ }^{71}$ Data from the clinical studies carried out with cefditoren in the treatment of pharyngotonsillitis from 2007 to 2010 in Japan showed that the percentage of adverse events was very low and diarrhea was the most frequent event. ${ }^{56-58}$ In the largest study (734 children), the incidence of adverse reactions was $1.50 \%$ (eleven events in eleven patients), with three events of diarrhea and three of hematuria in urinalysis without clinical symptoms. ${ }^{58}$ In a study carried out in children in Thailand comparing cefditoren-pivoxil (66 patients) with amoxicillin/ clavulanic acid ( 72 patients) for 10 days in the treatment of acute bacterial rhinosinusitis, the most frequent adverse event was diarrhea, with significant $(P=0.02)$ differences in the percentages found for both compounds $(4.5 \%$ with cefditorenpivoxil vs $18.1 \%$ for amoxicillin/clavulanic acid). ${ }^{69}$

Patients ingesting pivalic acid containing prodrugs may develop hypocarnitinemia. ${ }^{77}$ However, it has been reported that net carnitine losses after cefditoren-pivoxil administration are $<10 \%$ of body stores, thus it is not likely to result in adverse effects. ${ }^{78}$ Carnitine metabolism was studied in 16 pediatric patients under treatment with cefditoren-pivoxil, with increased carnitine excretion and a decrease in free carnitine in serum that disappeared when dosing was terminated without carnitine-related side effects. ${ }^{79}$ Nevertheless, two reports on clinical manifestations of hypocarnitinemia after cefditoren-pivoxil administration can be found in the literature: one reporting a carnitine-associated encephalopathy within hours of cefditoren-pivoxil administration for infection control after hysterectomy in a 47-years old woman $^{80}$ and the other in an 18-month child after 6 months of treatment for untreatable acute otitis media. ${ }^{81}$

\section{Optimizing cefditoren use}

The most prevalent respiratory infections in the community are pharyngotonsillitis, otitis media, sinusitis, AECB, and CAP, with $S$. pyogenes the most prevalent bacterial isolate from pharyngotonsillitis and S. pneumoniae and H. influenzae the most prevalent from the latter four infections. New antibiotics aiming to treat community respiratory infections should address the current issue of these bacterial species' resistance to common antibiotics.

As previously described, the main resistance issues in terms of $H$. influenzae is their resistance to macrolides in virtually all strains due to efflux pumps ${ }^{23}$ and the increasing prevalence of BLNAR and BLPACR phenotypes in certain geographical areas. ${ }^{27-29}$ These phenotypes/genotypes should be considered resistant to oral $\beta$-lactams such as amoxicillin, amoxicillin/clavulanic acid, cefaclor, and cefuroxime. ${ }^{82}$ Nontypable $H$. influenzae is one of the most common bacterial causes of AECB. ${ }^{83}$ It has been postulated that, with eradication, fewer viable pathogens remain in the bronchial tissue after antimicrobial treatment, requiring a longer period for the bacterial population to increase sufficiently to induce a new exacerbation, ${ }^{84}$ although the acquisition of new strains has also been associated with an increased risk of new exacerbations. ${ }^{85}$ Cefditoren may play an important therapeutic role in areas with significant rates of BLNAR/BLPACR phenotypes in the treatment of infections potentially caused by $H$. influenzae such as AECB.

During its clinical development, clinical trials of cefditoren in the treatment of lower respiratory tract infections showed its capability for eradication or presumed eradication of $S$. pneumoniae, including penicillin-intermediate and -resistant strains. ${ }^{75}$ Introduction of conjugate vaccines has produced a shift in serotype distribution (with their associated nonsusceptibility). There has been a decrease in PCV7 serotypes (associated with a decrease in penicillin/erythromycin nonsusceptibility), with an increase in non-PCV7 serotypes (some of them, as serotype 19A, non susceptible to penicillin). Although cefditoren exhibited an intrinsic activity higher than that of other $\beta$-lactams against these serotypes, ${ }^{86}$ there are no clinical studies showing the specific activity of cefditoren or other compounds against these emerging serotypes.

In addition, it has been pointed out that cefditoren may be the logical option for sequential therapy after intravenous treatment with cefotaxime or ceftriaxone for S. pneumoniae $\mathrm{CAP}^{87}$ This is based on cefditoren's spectrum and intrinsic in vitro activity, which is similar to that of intravenous third-generation cephalosporins (cefotaxime or ceftriaxone), together with its pharmacodynamic adequacy. ${ }^{87}$ 
Macrolide resistance is the main resistance issue in $S$. pyogenes, but strains are uniformly susceptible to all $\beta$-lactams. However, clinical failures of penicillin/amoxicillin treatment derived from indirect protection of S. pyogenes by $\beta$-lactamases produced by $M$. catarrhalis or $H$. influenzae have been reported ${ }^{88}$ Indirect protection of $S$. pyogenes could also affect amoxicillin/clavulanic acid efficacy when BLPACR strains are present, as has occurred in vitro. ${ }^{66}$ Oral $\beta$-lactams resistant to $\beta$-lactamases such as cefditoren could offer advantages by countering indirect pathogenicity, although clinical effects of indirect pathogenicity have been criticized by some authors. ${ }^{89,90}$

\section{Conclusion}

Cefditoren is a third-generation cephalosporin active against the principal community-acquired respiratory tract pathogens (including resistance phenotypes) that has demonstrated efficacy and safety in comparative clinical trials in adults with AECB, CAP, sinusitis, or pharyngotonsillitis.

The results of new clinical studies of cefditoren in the treatment of pharyngotonsillitis, sinusitis, and otitis media in children, mainly in Japan, support the adequacy of cefditoren for the treatment of these community-acquired respiratory tract infections and demonstrate that cefditoren has a safety profile similar to that of previous oral $\beta$-lactams. Cefditoren may be an interesting option in the pediatric field where fluoroquinolones are not indicated. Nevertheless, additional clinical trials in pediatric otitis media are needed, since penetration to middle-ear fluid is unknown.

Cefditoren is thus indicated for the treatment of mild-to-moderate community-acquired respiratory tract infections (when it is feasible for medication to be taken with meals) particularly in geographical areas with a reported prevalence of phenotypes exhibiting nonsusceptibility to common oral antibiotics among community respiratory pathogens.

\section{Disclosure}

LA and MJG have received travel grants from Tedec-Meiji Pharma to attend congresses for presentation of studies carried out on cefditoren. The authors declare no other conflicts of interest in this work.

\section{References}

1. World Health Organization, Department of Communicable Disease Surveillance and Response. WHO Global Strategy for Containment of Antimicrobial Resistance. Geneva: World Health Organization; 2001. Available from: http://www.who.int/csr/resources/publications/ drugresist/en/EGlobal_Strat.pdf. Accessed February 8, 2012.
2. Levy-Hara G, Amábile-Cuevas CF, Gould I, et al; for the International Society of Chemotherapy Antimicrobial Stewardship Working Group. "Ten Commandments" for the appropriate use of antibiotics by the practicing physician in an outpatient setting. Front Microbiol. 2011;2:230.

3. Livermore DM. Has the era of untreatable infections arrived? J Antimicrob Chemother. 2009;64(Suppl 1):i29-i36.

4. Mouton JW, Ambrose PG, Canton R, et al. Conserving antibiotics for the future: new ways to use old and new drugs from a pharmacokinetic and pharmacodynamic perspective. Drug Resist Updat. 2011;14(2):107-117.

5. Huovinen P, Cars O. Control of antimicrobial resistance: time for action. The essentials of control are already well known. BMJ. 1998; 317(7159):613-614

6. Lynch JP 3rd, Zhanel GG. Streptococcus pneumoniae: epidemiology and risk factors, evolution of antimicrobial resistance, and impact of vaccines. Curr Opin Pulm Med. 2010;16(3):217-225.

7. Fenoll A, Aguilar L, Granizo JJ, et al. Has the licensing of respiratory quinolones for adults and the 7-valent pneumococcal conjugate vaccine (PCV-7) for children had herd effects with respect to antimicrobial non-susceptibility in invasive Streptococcus pneumoniae? J Antimicrob Chemother. 2008;62(6):1430-1433.

8. Fenoll A, Granizo JJ, Aguilar L, et al. Temporal trends of invasive Streptococcus pneumoniae serotypes and antimicrobial resistance patterns in Spain from 1979 to 2007. J Clin Microbiol. 2009;47(4):1012-1020.

9. Fenoll A, Aguilar L, Vicioso MD, Gimenez MJ, Robledo O, Granizo JJ. Increase in serotype 19A prevalence and amoxicillin non-susceptibility among paediatric Streptococcus pneumoniae isolates from middle ear fluid in a passive laboratory-based surveillance in Spain, 1997-2009. BMC Infect Dis. 2011;11:239.

10. Farrell DJ, Couturier C, Hryniewicz W. Distribution and antibacterial susceptibility of macrolide resistance genotypes in Streptococcus pneumoniae: PROTEKT Year 5 (2003-2004). Int J Antimicrob Agents. 2008;31(3):245-249.

11. Lynch JP 3rd, Zhanel GG. Escalation of antimicrobial resistance among Streptococcus pneumoniae: implications for therapy. Semin Respir Crit Care Med. 2005;26(6):575-616.

12. Jones RN, Jacobs MR, Sader HS. Evolving trends in Streptococcus pneumoniae resistance: implications for therapy of community-acquired bacterial pneumonia. Int J Antimicrob Agents. 2010;36(3):197-204.

13. Granizo JJ, Aguilar L, Casal J, Dal-Ré R, Baquero F. Streptococcus pyogenes resistance to erythromycin in relation to macrolide consumption in Spain (1986-1997). J Antimicrob Chemother. 2000;46(6): 959-964.

14. Richter SS, Heilmann KP, Beekmann SE, et al. Macrolide-resistant Streptococcus pyogenes in the United States, 2002-2003. Clin Infect Dis. 2005;41(5):599-608.

15. Chan JC, Chu YW, Chu MY, Cheung TK, Lo JY. Epidemiological analysis of Streptococcus pyogenes infections in Hong Kong. Pathology. 2009;41(7):681-686.

16. Gracia M, Díaz C, Coronel P, et al. Antimicrobial susceptibility of Streptococcus pyogenes in Central, Eastern, and Baltic European Countries, 2005 to 2006: the cefditoren surveillance program. Diagn Microbiol Infect Dis. 2009;64(1):52-56.

17. Pérez-Trallero E, Martín-Herrero JE, Mazón A, et al; Spanish Surveillance Group for Respiratory Pathogens. Antimicrobial resistance among respiratory pathogens in Spain: latest data and changes over 11 years (1996-1997 to 2006-2007). Antimicrob Agents Chemother. 2010;54(7):2953-2959.

18. Yan SS, Fox ML, Holland SM, Stock F, Gill VJ, Fedorko DP. Resistance to multiple fluoroquinolones in a clinical isolate of Streptococcus pyogenes: identification of gyrA and parC and specification of point mutations associated with resistance. Antimicrob Agents Chemother. 2000;44(11):3196-3198.

19. Biedenbach DJ, Toleman MA, Walsh TR, Jones RN. Characterization of fluoroquinolone-resistant beta-hemolytic Streptococcus spp. isolated in North America and Europe including the first report of fluoroquinoloneresistant Streptococcus dysgalactiae subspecies equisimilis: report from the SENTRY Antimicrobial Surveillance Program (1997-2004). Diagn Microbiol Infect Dis. 2006;55(2):119-127. 
20. Richter SS, Diekema DJ, Heilmann KP, et al. Fluoroquinolone resistance in Streptococcus pyogenes. Clin Infect Dis. 2003;36(3):380-383.

21. Reinert RR, Lütticken R, Al-Lahham A. High-level fluoroquinolone resistance in a clinical Streptoccoccus pyogenes isolate in Germany. Clin Microbiol Infect. 2004;10(7):659-662.

22. Malhotra-Kumar S, Van Heirstraeten L, Lammens C, Chapelle S, Goossens H. Emergence of high-level fluoroquinolone resistance in emm6 Streptococcus pyogenes and in vitro resistance selection with ciprofloxacin, levofloxacin and moxifloxacin. JAntimicrob Chemother. 2009;63(5):886-894.

23. Tristram S, Jacobs MR, Appelbaum PC. Antimicrobial resistance in Haemophilus influenzae. Clin Microbiol Rev. 2007;20(2):368-389.

24. Sahm DF, Brown NP, Thornsberry C, Jones ME. Antimicrobial susceptibility profiles among common respiratory tract pathogens: a GLOBAL perspective. Postgrad Med. 2008;120(3 Suppl 1):16-24.

25. Sahm DF, Brown NP, Draghi DC, Evangelista AT, Yee YC, Thornsberry C. Tracking resistance among bacterial respiratory tract pathogens: summary of findings of the TRUST Surveillance Initiative, 2001-2005. Postgrad Med. 2008;120(3 Suppl 1):8-15.

26. Jansen WT, Verel A, Beitsma M, Verhoef J, Milatovic D. Longitudinal European surveillance study of antibiotic resistance of Haemophilus influenzae. J Antimicrob Chemother. 2006;58(4):873-877.

27. Jansen WT, Verel A, Beitsma M, Verhoef J, Milatovic D. Surveillance study of the susceptibility of Haemophilus influenzae to various antibacterial agents in Europe and Canada. Curr Med Res Opin. 2008;24(10):2853-2861.

28. Kishii K, Chiba N, Morozumi M, et al. Diverse mutations in the ftsI gene in ampicillin-resistant Haemophilus influenzae isolates from pediatric patients with acute otitis media. $J$ Infect Chemother. 2010;16(2):87-93.

29. Ito M, Hotomi M, Maruyama Y, et al. Clonal spread of beta-lactamaseproducing amoxicillin-clavulanate-resistant (BLPACR) strains of nontypeable Haemophilus influenzae among young children attending a day care in Japan. Int J Pediatr Otorhinolaryngol. 2010;74(8):901-906.

30. Suzuki K, Nishimaki K, Okuyama K, et al. Trends in antimicrobial susceptibility of in the Tohoku district of Japan: a longitudinal analysis from 1998 to 2007. Tohoku J Exp Med. 2010;220(1):47-57.

31. Kang JH, Lee SY, Kim JH, Hur JK, Lee KY. In vitro antimicrobial activity of cefditoren and other oral antibiotics against Streptococcus pneumoniae, isolated from children with community acquired respiratory tract infections. Jpn J Antibiot. 2010;63(1):11-17.

32. Biedenbach DJ, Jones RN. Update of cefditoren activity tested against community-acquired pathogens associated with infections of the respiratory tract and skin and skin structures, including recent pharmacodynamic considerations. Diagn Microbiol Infect Dis. 2009;64(2):202-212.

33. Fritsche TR, Biedenbach DJ, Jones RN. Update of the activity of cefditoren and comparator oral beta-lactam agents tested against community-acquired Streptococcus pneumoniae isolates (USA, 2004-2006). J Chemother. 2008;20(2):170-174.

34. Stefani S, Mezzatesta ML, Fadda G, et al. Antibacterial activity of cefditoren against major community-acquired respiratory pathogens recently isolated in Italy. $J$ Chemother. 2008;20(5):561-569.

35. Tempera G, Furneri PM, Ferranti C, et al. In vitro activity of cefditoren versus other antibiotics against $\mathrm{S}$. pneumoniae clinical strains isolated in Italy. Int J Immunopathol Pharmacol. 2010;23(3):833-840.

36. Tempera G, Furneri PM, Carlone NA, et al. Antibiotic susceptibility of respiratory pathogens recently isolated in Italy: focus on cefditoren. J Chemother. 2010;22(3):153-159.

37. Seral C, Suárez L, Rubio-Calvo C, et al. In vitro activity of cefditoren and other antimicrobial agents against 288 Streptococcus pneumoniae and 220 Haemophilus influenzae clinical strains isolated in Zaragoza, Spain. Diagn Microbiol Infect Dis. 2008;62(2):210-215.

38. Fenoll A, Giménez MJ, Robledo O, et al. Activity of cefditoren against clinical isolates of Streptococcus pneumoniae showing nonsusceptibility to penicillins, cephalosporins, macrolides, ketolides or quinolones. Int J Antimicrob Agents. 2007;29(2):224-226.
39. Fenoll A, Aguilar L, Robledo O, et al. Influence of the $\beta$-lactam resistance phenotype on the cefuroxime versus cefditoren susceptibility of Streptococcus pneumoniae and Haemophilus influenzae recovered from children with acute otitis media. J Antimicrob Chemother. 2007;60(2):323-327.

40. Fenoll A, Giménez MJ, Robledo O, et al. Influence of penicillin/ amoxicillin non-susceptibility on the activity of third-generation cephalosporins against Streptococcus pneumoniae. Eur JClin Microbiol Infect Dis. 2008;27(1):75-80.

41. Fenoll A, Giménez MJ, Robledo O, et al. In vitro activity of oral cephalosporins against pediatric isolates of Streptococcus pneumoniae non-susceptible to penicillin, amoxicillin or erythromycin. JChemother. 2008;20(2):175-179.

42. Kozlov RS, Sivaya OV, Shevelev AN. Perspective of new cephalosporins for treatment of pneumococcal infections. Pulmonology. 2011;3:53-58. Article in Russian, htpp://www.pulmonology.ru.

43. Sevillano D, Giménez MJ, Cercenado E, et al. Genotypic versus phenotypic characterization, with respect to beta-lactam susceptibility, of Haemophilus influenzae isolates exhibiting decreased susceptibility to beta-lactam resistance markers. Antimicrob Agents Chemother. 2009;53(1):267-270.

44. Gracia M, Díaz C, Coronel P, et al. Antimicrobial susceptibility of Haemophilus influenzae and Moraxella catarrhalis isolates in eight Central, East and Baltic European countries in 2005-2006: results of the Cefditoren Surveillance Study. J Antimicrob Chemother. 2008;61(5):1180-1181.

45. Harimaya A, Yokota S, Sato K, Himi T, Fujii N. Remarkably high prevalence of fts I gene mutations in Haemophilus influenzae isolates from upper respiratory tract infections in children of the Sapporo district, Japan. J Infect Chemother. 2008;14(3):223-227.

46. García-de-Lomas J, Lerma M, Cebrián L, et al. Influence of Haemophilus influenzae beta-lactamase production and/or ftsI gene mutations on in vitro activity of and susceptibility rates to aminopenicillins and second- and third-generation cephalosporins. Int J Antimicrob Agents. 2007;30(2):190-192.

47. Biedenbach DJ, Jones RN, Fritsche TR. Antimicrobial activity of cefditoren tested against contemporary (2004-2006) isolates of Haemophilus influenzae and Moraxella catarrhalis responsible for community-acquired respiratory tract infections in the United States. Diagn Microbiol Infect Dis. 2008;61(2):240-244.

48. Wellington K, Curran MP. Cefditoren pivoxil: a review of its use in the treatment of bacterial infections. Drugs. 2004;64(22): 2597-2618

49. Sádaba B, Azanza JR, Quetglas EG, et al. Pharmacokinetic/pharmacodynamic serum and urine profile of cefditoren following single-dose and multiple twice- and thrice-daily regimens in healthy volunteers: a phase I study. Rev Esp Quimioter. 2007;20(1):51-60.

50. Lodise TP, Kinzig-Schippers M, Drusano GL, et al. Use of population pharmacokinetic modeling and Monte Carlo simulation to describe the pharmacodynamic profile of cefditoren in plasma and epithelial lining fluid. Antimicrob Agents Chemother. 2008;52(6): 1945-1951.

51. Craig WA. Pharmacokinetic/pharmacodynamic parameters: rationale for antibacterial dosing of mice and men. Clin Infect Dis. 1998;26(1):1-10.

52. Heffelfinger JD, Dowell SF, Jorgensen JH, et al. Management of community-acquired pneumonia in the era of pneumococcal resistance: a report from the Drug-Resistant Streptococcus pneumoniae Therapeutic Working Group. Arch Intern Med. 2000;160(10): 1399-1408.

53. Granizo JJ, Sádaba B, Honorato J, et al. Monte Carlo simulation describing the pharmacodynamic profile of cefditoren in plasma from healthy volunteers. Int J Antimicrob Agents. 2008;31(4):396-398.

54. Cafini F, Yuste J, Giménez MJ, et al. Enhanced in vivo activity of cefditoren in pre-immunized mice against penicillin-resistant $\mathrm{S}$. pneumoniae (serotypes 6B, 19F and 23F) in a sepsis model. PLoS One. 2010; 5(8):e12041. 
55. Tsumura N, Nagai K, Hidaka $H$, et al. Antibiotic therapy against acute tonsillopharyngitis in children due to group A beta-hemolytic streptococci: comparison of clinical efficacy, the bactericidal effects, and effects on oral flora between cefditoren pivoxil for 5 days and amoxicillin for 10 days. Jpn J Antibiot. 2011;64(3):179-190. Japanese.

56. Ozaki T, Nishimura N, Suzuki M, et al. Five-day oral cefditoren pivoxil versus 10-day oral amoxicillin for pediatric group A streptococcal pharyngotonsillitis. J Infect Chemother. 2008;14(3):213-218.

57. Sakata H. A multicenter study of 7-day cefditoren pivoxil treatment for group A streptococcal infection. Jpn J Antibiot. 2007;60(5):257-263. Japanese.

58. Kawamata S, Yamada H, Sato Y, Sasagawa Y, Iwama Y, Matumoto M. Evaluation of the safety and efficacy of cefditoren pivoxil fine granules for pediatric use in pediatric patients with laryngopharyngitis and tonsillitis caused by Streptococcus pyogenes. Jpn JAntibiot. 2010;63(4): 299-311. Japanese.

59. Ogawa T, Terao Y, Okuni H, et al. Biofilm formation or internalization into epithelial cells enable Streptococcus pyogenes to evade antibiotic eradication in patients with pharyngitis. Microb Pathog. 2011; 51(1-2):58-68.

60. Kaplan EL, Tucker RM, Poling TL, Marsh D, Chow C. Managing group A streptococcal infection. A multicenter comparison of cefditoren pivoxil and penicillin VK. J Resp Dis. 2001;22(Suppl 8): S60-S64.

61. Gooch W, Marsh D, Slickler T, Hunt B. Cefditoren is safe and effective treatment for streptococcal pharyngitis. In: Abstracts of the 40th Interscience Conference on Antimicrobial Agents and Chemotherapy, Toronto 2000. Abstract 837. Washington DC: American Society for Microbiology.

62. Granizo JJ, Giménez MJ, Barberán J, Coronel P, Gimeno M, Aguilar L. Efficacy of cefditoren in the treatment of upper respiratory tract infections: a pooled analysis of six clinical trials. Rev Esp Quimioter. 2008;21(1):14-21.

63. Chole RA, Faddis BT. Anatomical evidence of microbial biofilms in tonsillar tissues: a possible mechanism to explain chronicity. Arch Otolaryngol Head Neck Surg. 2003;129(6):634-636.

64. Swidsinski A, Göktas O, Bessler C, et al. Spatial organisation of microbiota in quiescent adenoiditis and tonsillitis. $J$ Clin Pathol. 2007;60(3):253-260.

65. Galli J, Calò L, Ardito F, et al. Biofilm formation by Haemophilus influenzae isolated from adeno-tonsil tissue samples, and its role in recurrent adenotonsillitis. Acta Otorhinolaryngol Ital. 2007;27(3): 134-138.

66. Sevillano D, Aguilar L, Alou L, et al. Beta-lactam effects on mixed cultures of common respiratory isolates as an approach to treatment effects on nasopharyngeal bacterial population dynamics. PLoS One. 2008;3(12): e3846.

67. Pichichero ME, Casey JR. Systematic review of factors contributing to penicillin treatment failure in Streptococcus pyogenes pharyngitis. Otolaryngol Head Neck Surg. 2007;137(6):851-857.

68. Kikuta H, Shibata M, Nakata S, et al. Comparative study of 5-day and 10-day cefditoren pivoxil treatments for recurrent group A beta-hemolytic Streptococcus pharyngitis in children. Int J Pediatr. 2009;2009:863608.

69. Poachanukoon O, Kitcharoensakkul M. Efficacy of cefditoren pivoxil and amoxicillin/clavulanate in the treatment of pediatric patients with acute bacterial rhinosinusitis in Thailand: a randomized, investigatorblinded, controlled trial. Clin Ther. 2008;30(10):1870-1879.

70. Chow J, Russell M, Volk S, Chow C. Efficacy of cefditoren pivoxil (CDTR) versus amoxicillin/clavulanate (AMX/CLV) in acute maxillary sinusitis (AMS). In: Abstracts of the 40th Interscience Conference on Antimicrobial Agents and Chemotherapy, Toronto 2000. Abstract 835. Washington DC: American Society for Microbiology.

71. Kawamata S, Yamada H, Sato Y, Sasagawa Y, Iwama Y, Matumoto M. Evaluation of the safety and efficacy of cefditoren pivoxil fine granules for pediatric use in pediatric patients with acute otitis media. Jpn J Antibiot. 2010;63(3):207-223. Japanese.
72. Sakata H. Comparative study on bacterial eradication rate and clinical efficacy of CDTR, CFPN, and FRPM for treatment of children with otitis media and lower respiratory tract infection due to Streptococcus pneumoniae and Haemophilus influenzae. Jpn J Antibiot. 2001; 54 Suppl B:96. Japanese.

73. Ito M, Furukawa M. High-dose cefditren therapy for children with acute otitis media that had Streptococcus pneumoniae and Haemophilus influenzae isolated from the nasopharynx. Jpn J Antibiot. 2001; 54 Suppl B:37-38. Japanese

74. Sugita R, Deguchi K, Kimura S, et al. Efficacy of cefditoren pivoxil in the treatment of acute otitis media due to benzylpenicillin-insensitive Streptococcus pneumoniae. Jpn J Antibiot. 1996;49(4):386-398. Japanese.

75. Granizo JJ, Giménez MJ, Barberán J, Coronel P, Gimeno M, Aguilar L. The efficacy of cefditoren pivoxil in the treatment of lower respiratory tract infections, with a focus on the per-pathogen bacteriologic response in infections caused by Streptococcus pneumoniae and Haemophilus influenzae: a pooled analysis of seven clinical trials. Clin Ther. 2006; 28(12):2061-2069.

76. Granizo JJ, Aguilar L, Gimenez MJ, Coronel P, Gimeno M, Prieto J. Safety profile of cefditoren. A pooled analysis of data from clinical trials in community-acquired respiratory tract infections. Rev Esp Quimioter. 2009;22(2):57-61.

77. Todesco L, Bodmer M, Vonwil K, Häussinger D, Krähenbühl S. Interaction between pivaloylcarnitine and L-carnitine transport into L6 cells overexpressing hOCTN2. Chem Biol Interact. 2009;180(3): 472-477.

78. Brass EP, Mayer MD, Mulford DJ, Stickler TK, Hoppel CL. Impact on carnitine homeostasis of short-term treatment with the pivalate prodrug cefditoren pivoxil. Clin Pharmacol Ther. 2003;73(4):338-347.

79. Fujii R, Chiba S, Numazaki K, et al. Effect of cefditoren pivoxil on carnitine metabolism in pediatric patients. Jpn J Antibiot. 1993; 46(10):926-937. Erratum in: Jpn J Antibiot. 1994;47(9):1240. Japanese.

80. Kim H, Chu K, Jung KH, Lee ST, Kim JM, Lee SK. Acquired encephalopathy associated with carnitine deficiency after cefditoren pivoxil administration. Neurol Sci. 2012. Epub Jan 19.

81. Makino Y, Sugiura T, Ito T, Sugiyama N, Koyama N. Carnitine-associated encephalopathy caused by long-term treatment with an antibiotic containing pivalic acid. Pediatrics. 2007;120(3):e739-e741.

82. Clinical and Laboratory Standards Institute. Performance standards for antimicrobial susceptibility testing; nineteenth informational supplement. CLSI document M100-S19. Clinical and Laboratory Standards Institute, Wayne, PA, USA, 2009.

83. Murphy TF. The role of bacteria in airway inflammation in exacerbations of chronic obstructive pulmonary disease. Curr Opin Infect Dis. 2006;19(3):225-230.

84. Chodosh S. Clinical significance of the infection-free interval in the management of acute bacterial exacerbations of chronic bronchitis. Chest. 2005;127(6):2231-2236.

85. Sethi S, Evans N, Grant BJ, Murphy TF. New strains of bacteria and exacerbations of chronic obstructive pulmonary disease. $N$ Engl J Med. 2002;347(7):465-471.

86. Fenoll A, Aguilar L, Giménez MJ, et al. Susceptibility of recently collected Spanish pneumococci nonsusceptible to oral penicillin from serotypes not included in the 7-valent conjugate vaccine. Antimicrob Agents Chemother. 2010;54(6):2696-2698.

87. Barberán J, Mensa J. Cefditoren and community-acquired lower respiratory tract infections (corrected). Rev Esp Quimioter. 2009;22(3): 144-150. Spanish.

88. Casey JR, Pichichero ME. The evidence base for cephalosporin superiority over penicillin in streptococcal pharyngitis. Diagn Microbiol Infect Dis. 2007;57(Suppl 3):39S-45S.

89. Bisno AL. Are cephalosporins superior to penicillin for treatment of acute streptococcal pharyngitis? Clin Infect Dis. 2004;38:1535-1537.

90. Shulman ST, Gerber MA. So what's wrong with penicillin for strep throat? Pediatrics. 2004;113(6):1816-1819. 


\section{Publish your work in this journal}

The International Journal of General Medicine is an international, peer-reviewed open-access journal that focuses on general and internal medicine, pathogenesis, epidemiology, diagnosis, monitoring and treatment protocols. The journal is characterized by the rapid reporting of reviews, original research and clinical studies across all disease areas.

A key focus is the elucidation of disease processes and management protocols resulting in improved outcomes for the patient.The manuscript management system is completely online and includes a very quick and fair peer-review system. Visit http://www.dovepress.com/ testimonials.php to read real quotes from published authors.

Submit your manuscript here: http://www.dovepress.com/international-journal-of-general-medicine-journal 